\title{
Dietary Estimated Intake of Trace Elements: Risk Assessment in an Italian Population
}

\author{
Tommaso Filippini ${ }^{1}$ (1) $\cdot$ Stefano Tancredi ${ }^{1} \cdot$ Carlotta Malagoli $^{1} \cdot$ Marcella Malavolti $^{1} \cdot$ Annalisa Bargellini $^{1}$. \\ Luciano Vescovi ${ }^{1} \cdot$ Fausto Nicolini ${ }^{2} \cdot$ Marco Vinceti $^{1,3}$
}

Received: 23 August 2019 / Revised: 14 September 2019 / Accepted: 21 September 2019 / Published online: 9 October 2019

(c) The Author(s) 2019

\begin{abstract}
Dietary intake to trace elements may represent the most relevant source of exposure for the general, non-occupationally population, but some of them have been rarely evaluated. We measured content of fifteen trace elements (antimony, barium, beryllium, boron, cobalt, lithium, molybdenum, nickel, silver, strontium, tellurium, thallium, titanium, uranium, and vanadium) in 908 food and beverage samples through inductively coupled plasma mass spectrometry. We estimated their dietary intake using a validated semi-quantitative food frequency questionnaire collected from a population of the Emilia-Romagna Region in Northern Italy. We compared our estimates with tolerable upper intake levels reported by international agencies and we assessed the non-carcinogenic risk through calculation of total hazard quotient for each trace element according to the US-EPA approach. Overall, estimates of their dietary intake were substantially similar to those reported from other countries, and they fell below the tolerable upper intake levels provided by international agencies. The total hazard quotient for each trace element was below 1 . Our findings provide updated estimates of food levels and dietary intake of trace elements far frequently evaluated in a sample of Italian adult consumers. They also suggest that any non-carcinogenic risk associated with intake of investigated trace elements may be ruled out in our population.
\end{abstract}

Keywords Trace elements $\cdot$ Dietary intake $\cdot$ Food contamination $\cdot$ Food safety $\cdot$ Risk assessment

\section{Introduction}

A comprehensive assessment of dietary intake of chemical contaminants is needed to evaluate the long-term risk for public health and food risk assessment (European Food Safety Authority 2006; US-EPA; WHO 1996), considering that diet represents the most relevant source of majority of

Electronic supplementary material The online version of this article (https://doi.org/10.1007/s12403-019-00324-w) contains supplementary material, which is available to authorized users.

Tommaso Filippini

tommaso.filippini@unimore.it

1 Environmental, Genetic and Nutritional Epidemiology Research Center (CREAGEN), Section of Public Health, Department of Biomedical, Metabolic and Neural Sciences, University of Modena and Reggio Emilia, Via Campi 287, 41125 Modena, Italy

2 Local Health Authority, Reggio Emilia, Italy

3 Department of Epidemiology, Boston University School of Public Health, Boston, USA trace elements for non-occupationally exposed populations (Reilly 2002). In order to assess the possible health risk to the consumers, it seems necessary to evaluate trace element content in foods and beverages that are consumed by the general population and to estimate their actual dietary intake for comparison with tolerable levels (European Food Safety Authority 2006). In recent years, a few studies assessed the levels of rare or 'neglected' trace elements, especially in the Italian population (Filippini et al. 2018a; Turconi et al. 2009), and for some elements results are lacking also for Europe and other Western population (European Food Safety Authority 2011; Reilly 2002). In addition, the relevance of trace elements in human health and disease is well documented. Depending also of their role within the metabolism, they present an intriguing relation with human health, showing either nutritional and toxicological effects (Nordberg and Nordberg 2016; WHO 1996). Being diet most relevant source of exposure to the above-mentioned trace elements, a periodic and updated evaluation of their content in foods represents a key element for a comprehensive assessment of 
their intake levels and of possible consequent health risks (Kim et al. 2015).

To do this, in this study, we aimed to estimate the dietary intake of a large number of trace elements (namely antimony, barium, beryllium, boron, cobalt, lithium, molybdenum, nickel, silver, strontium, tellurium, thallium, titanium, uranium, and vanadium) in a Northern Italy population, in order to assess their exposure level through diet and evaluate whether these levels are safe for human health.

\section{Methods}

\section{Food Collection and Analysis}

We determined the content of trace elements in food samples available and collected in the area of the study. Food collection lasted from October 2016 to February 2017. We purchased a total of 908 food and beverage samples in local markets, large supermarkets, and grocery stores as well as in community canteens from Reggio Emilia and Modena provinces. Relevant food items characterizing the diet of this community were selected from previous populationbased studies addressing the dietary habits of subjects from Northern Italy, with particular reference of Emilia-Romagna Region (Filippini et al. 2018a). In order to avoid cross contamination with metals and trace element under investigation, we used plastic food containers (i.e. $50 \mathrm{ml}$ volume plastic tubes or jars) as well as plastic cutlery or stainless knives when handling and collecting food samples. Using a clean stainless-steel knife, we cut solid foods by collecting samples from six different points in the plate. Then, we homogenized the samples using a food blender equipped with a stainless-steel blade and we placed a portion of $0.5 \mathrm{~g}$ in quartz containers previously washed with MilliQ water (MilliQPlus, Millipore, MA, USA) and $\mathrm{HNO}_{3}$. We liquid-ashed the samples with $10 \mathrm{ml}$ solution $\left(5 \mathrm{ml} \mathrm{HNO}_{3}+5 \mathrm{ml} \cdot \mathrm{H}_{2} \mathrm{O}\right)$ in a microwave digestion system (Discover SP-D, CEM Corporation, NC, USA) and we finally stored them in plastic tubes, and diluted to $50 \mathrm{ml}$ with deionized water before analysis. Using an inductively coupled plasma mass spectrometer (Agilent 7500ce, Agilent Technologies, CA, USA), we performed trace element determination. All the analyses were performed in duplicate, implementing quality controls including both blanks (solution of MilliQ water) and a control solution of tap water additionally enriched with $22 \mathrm{ppb}$ of each element under investigation (Filippini et al. 2018b, 2019b). Limits of detection (LOD) are presented in Table 1 and values below the LOD were set equal to LOD/2.

We report the contamination levels of selected trace elements according to the food consumption patterns and food categories typical of this Italian population, as assessed through the European Prospective Investigation into Cancer
Table 1 Limit of quantification (LOQ) and limit of detection (LOD) of investigated elements

\begin{tabular}{lll}
\hline Element & LOQ & LOD \\
\hline Antimony (Sb) & 0.00390 & 0.00130 \\
Barium (Ba) & 0.79092 & 0.26364 \\
Beryllium (Be) & 0.00012 & 0.00004 \\
Boron (B) & 0.95640 & 0.31880 \\
Cobalt (Cb) & 0.04956 & 0.01652 \\
Lithium (Lt) & 0.02322 & 0.00774 \\
Molybdenum (Mo) & 0.05424 & 0.01808 \\
Nickel (Ni) & 0.31800 & 0.10600 \\
Silver (Ag) & 0.00012 & 0.00004 \\
Strontium (Sr) & 0.54096 & 0.18032 \\
Tellurium (Te) & 0.00210 & 0.00070 \\
Thallium (Tl) & 0.00300 & 0.00100 \\
Titanium (Ti) & 0.43914 & 0.14638 \\
Uranium (U) & 0.00096 & 0.00032 \\
Vanadium (V) & 0.05298 & 0.01766 \\
\hline
\end{tabular}

All values are in $\mu \mathrm{g} / \mathrm{kg}$

and Nutrition-EPIC food frequency questionnaire implemented for dietary habits evaluation (Filippini et al. 2017a, 2017b; Turrini et al. 2001). The final list of main food categories includes cereals and cereal products, meat and meat products, milk and dairy products, eggs, fish and seafood, vegetables, legumes, potatoes, fresh fruits, dry fruits, sweets, and beverages.

\section{Study Population and Assessment of Dietary Habits}

We assessed dietary habits in sample population in EmiliaRomagna Region, Northern Italy. Detailed information of participant identification and recruitment has been previously reported in detail (Malavolti et al. 2013; Malavolti et al. 2017). To sum up, after the approval of the provincial ethical committee, and through the access to the National Health Service directory including all residents of the Emilia-Romagna Region, namely from the provinces of Bologna, Ferrara, Modena, Parma, and Reggio Emilia, we invited to participate to the study 2825 subjects, and eventually $747(26.4 \%)$ agreed to participate and provided their written consent. The participants returned both a lifestyle questionnaire and the EPIC food frequency questionnaire (FFQ) which we had mailed to them. The EPIC FFQ is a validated semi-quantitative FFQ specifically developed for the Central-Northern Italy population (Pala et al. 2003; Pasanisi et al. 2002) within the 'European Prospective Investigation into Cancer and Nutrition' project. This FFQ was designed to estimate frequency and amount of consumption of 188 food items over the previous year, also using photos of serving sizes to help proper completion by participants. 
Out of those who returned study materials, we excluded twenty-eight subjects from subsequent analysis because of incomplete data or extreme and implausible values of energy intake derived from the FFQ (assessed though the ratio of total energy intake:calculated basal metabolic rate lower than the 0.5 th percentile or higher than the 99.5 th percentile). The final population sample comprised 719 (men/women 319/400) adult subjects, with age ranging from 18 to 87 years, with 499 subjects (male/female 190/309) aged $<65$ years and 220 (male/female 129/91) aged $\geq 65$ years. Mean age was 55.2 (standard deviation 14.5) years in overall sample with slightly higher values in men than women with mean age of 59.1 (14.0) year and 52.3 (14.1) years, respectively. (Filippini et al. 2019a; Malagoli et al. 2019). The median energy intake was $1906 \mathrm{kcal} /$ day (interquartile range-IQR $1538-2364 \mathrm{kcal} /$ day) in all subjects, $2024 \mathrm{kcal} /$ day (IQR 1649-2462 kcal/day) in men and $1800 \mathrm{kcal} /$ day (IQR $1455-2296 \mathrm{kcal} / \mathrm{day}$ ) in women, respectively.

\section{Dietary Intake Estimates of Trace Elements}

We combined data from the determination of contamination levels of trace elements in foods and the dietary habits assessed using the FFQ to compute trace element daily intake, by using the equation presented below.

$$
\begin{aligned}
& \text { Daily dietary estimate }\left(\frac{\mu \mathrm{g}}{\text { day }}\right) \\
& =\sum \frac{\text { level in food }\left(\frac{\mu \mathrm{g}}{\mathrm{kg}}\right) \times \text { food intake }\left(\frac{\mathrm{g}}{\text { day }}\right)}{1000}
\end{aligned}
$$

We multiplied the element contents measured in food $(\mu \mathrm{g} /$ $\mathrm{kg}$ ) with the intake as estimated by the FFQ (g/day). Accordingly, we estimated the daily dietary intake of the rare trace elements for the diet as a whole and for each food category, by reporting median and interquartile ranges (IQR). We also estimated the dietary intake by kilogram $(\mathrm{kg})$ of body weight (bw), by dividing for weight of participants.

Finally, we implemented the human health risk assessment method as suggested by the US-Environmental Protection Agency (US-EPA) in order to evaluate the probability of adverse health effects in humans exposed to selected trace element through diet (Adel et al. 2016; Bonsignore et al. 2018; Copat et al. 2018; Ferrante et al. 2018). In particular, the safety risk assessment using target hazard quotient (THQ) promoted by US-EPA describes the non-cancer risk of contaminants by the ratio between exposure dose (i.e. dietary intake) and the reference dose (RfD) (US-EPA). Detailed expression is as follows:

$$
\sum \mathrm{THQ}=\sum \frac{\mathrm{DI}}{\mathrm{RfD}}
$$

where THQ is the target hazard quotient, DI is the dietary daily intake in $\mu \mathrm{g} / \mathrm{kg}$ bw/day, and RfD is the reference dose in $\mu \mathrm{g} / \mathrm{kg}$ bw/day. A THQ $<1$ indicates that the trace element exposure level is no harmful, while a THQ $>1$ indicates that it has potential harm to the human body (US-EPA).

\section{Results and Discussion}

Only a few elements show values below the LOD of a large amount of food (Supplemental Table S1), in particular silver (48.6\%), thallium (35.8\%), tellurium (22.2\%), and partially beryllium (14.1\%) and antimony (11.0\%). Tables 2 and 3 present the levels of trace element contamination in foods and the estimates of their dietary intake according to the main food categories, respectively, showing their median and interquartile range. Corresponding figures according to subcategories are presented in Supplemental Tables S2-S3, and dietary estimates in subjects aged $<65$ and $\geq 65$ years are presented in Supplemental Tables S4 and S5, respectively. Table 4 compares the estimated median and the highest level we estimated in study population with the tolerable intake suggested by international regulatory agencies. Detailed results are presented for each trace element separately. Overall, our findings are similar compared to data on dietary intake reported in other countries when available.

\section{Antimony}

Antimony occurs mainly in a trivalent (as antimony trioxide) or pentavalent state (antimony potassium tartrate) (WHO 2003b). Antimony trioxide is considered a food contaminant due to its use as additive and initiator in the manufacture of polyethylene terephthalate and other polymers. Food contamination follows migration from food contact materials in which they are used (ECHA 2008; European Food Safety Authority 2004b). Antimony is also a naturally occurring element, therefore, its presence in the environment, and thereby also indirectly in water and in foods and beverages produced from agricultural goods, may also be attributed to natural sources (WHO 2003b). Antimony shows higher concentration in sweets products, followed by meat and fish (Table 2). Foods showing the highest antimony content are biscuits, dry cakes, chocolate, and candy bars among sweet products, but also mushrooms, processed meat, crustaceans, molluscs, and preserved and tinned fish demonstrated concentration above $2 \mu \mathrm{g} / \mathrm{kg}$ (Supplemental Table S2). The antimony daily dietary intake is $3.471 \mu \mathrm{g} /$ day (IQR 2.801-4.395 $\mu \mathrm{g} /$ day) (Table 3), with main contribution from fresh fruits (particularly citrus fruits), cereal products (mainly bread), and meat (red and processed ones) (Supplemental Table S3). Our results are consistent with previous 
Table 2 Trace element concentrations in main food categories

\begin{tabular}{|c|c|c|c|c|c|c|}
\hline \multirow[t]{2}{*}{ Food category } & \multirow[b]{2}{*}{50 th } & \multicolumn{2}{|l|}{ Antimony $(\mu \mathrm{g} / \mathrm{kg})$} & \multicolumn{2}{|l|}{ Barium $(\mathrm{mg} / \mathrm{kg})$} & \multirow{2}{*}{$\begin{array}{l}\text { Beryllium }(\mu \mathrm{g} / \mathrm{kg}) \\
(\mathrm{IQR})\end{array}$} \\
\hline & & (IQR) & 50 th & (IQR) & 50 th & \\
\hline Cereals and cereal products & 1.361 & $(0.263-3.438)$ & 1.034 & $(0.724-1.400)$ & 0.135 & $(0.033-0.269)$ \\
\hline Meat and meat products & 2.025 & $(0.836-4.284)$ & 0.038 & $(0.022-0.072)$ & 0.042 & $(0.007-0.106)$ \\
\hline Milk and dairy products & 1.031 & $(0.294-2.800)$ & 0.539 & $(0.125-0.801)$ & 0.048 & $(0.005-0.113)$ \\
\hline Eggs & 0.756 & $(0.158-0.941)$ & 0.214 & $(0.031-1.096)$ & 0.001 & $(0.000-0.048)$ \\
\hline Fish and seafood & 1.838 & $(0.551-3.783)$ & 0.056 & $(0.026-0.165)$ & 0.077 & $(0.024-0.226)$ \\
\hline All vegetables & 1.179 & $(0.276-3.897)$ & 0.217 & $(0.081-0.532)$ & 0.082 & $(0.020-0.220)$ \\
\hline Legumes & 1.185 & $(0.001-2.698)$ & 0.893 & $(0.519-2.010)$ & 0.342 & $(0.146-0.578)$ \\
\hline Potatoes & 1.489 & $(0.170-3.468)$ & 0.088 & $(0.065-0.174)$ & 0.109 & $(0.019-0.376)$ \\
\hline Fresh fruits & 0.526 & $(0.097-2.070)$ & 0.169 & $(0.081-0.344)$ & 0.059 & $(0.006-0.174)$ \\
\hline Dry fruits, nuts, and seeds & 1.535 & $(0.634-4.403)$ & 0.927 & $(0.568-2.445)$ & 0.277 & $(0.037-0.355)$ \\
\hline Sweets, chocolate, cakes, etc. & 2.101 & $(1.086-5.842)$ & 0.693 & $(0.269-2.145)$ & 0.145 & $(0.031-0.333)$ \\
\hline Oils and fats & 0.352 & $(0.150-1.285)$ & 0.007 & $(0.002-0.015)$ & 0.003 & $(0.000-0.022)$ \\
\hline \multirow[t]{2}{*}{ Beverages } & 0.363 & $(0.124-0.745)$ & 0.039 & $(0.014-0.087)$ & 0.067 & $(0.013-0.280)$ \\
\hline & 50 th & $\begin{array}{l}\text { Boron }(\mathrm{mg} / \mathrm{kg}) \\
\text { (IQR) }\end{array}$ & 50 th & $\begin{array}{l}\text { Cobalt }(\mu \mathrm{g} / \mathrm{kg}) \\
(\mathrm{IQR})\end{array}$ & 50 th & $\begin{array}{l}\text { Lithium }(\mu \mathrm{g} / \mathrm{kg}) \\
(\mathrm{IQR})\end{array}$ \\
\hline Cereals and cereal products & 0.368 & $(0.188-0.751)$ & 7.08 & $(4.03-13.11)$ & 14.83 & $(7.16-29.15)$ \\
\hline Meat and meat products & 0.143 & $(0.059-0.256)$ & 2.87 & $(2.01-5.52)$ & 3.41 & $(2.01-6.05)$ \\
\hline Milk and dairy products & 0.164 & $(0.081-0.266)$ & 6.88 & $(3.60-12.24)$ & 4.78 & $(2.75-9.43)$ \\
\hline Eggs & 0.202 & $(0.181-0.331)$ & 2.49 & $(0.94-5.48)$ & 3.87 & $(1.50-6.51)$ \\
\hline Fish and seafood & 0.306 & $(0.097-0.554)$ & 4.91 & $(2.98-7.45)$ & 19.10 & $(10.49-38.80)$ \\
\hline All vegetables & 1.415 & $(0.956-2.427)$ & 6.05 & $(3.71-13.92)$ & 8.23 & $(2.37-23.26)$ \\
\hline Legumes & 7.756 & $(3.537-11.090)$ & 72.84 & $(39.28-104.29)$ & 15.43 & $(6.19-35.66)$ \\
\hline Potatoes & 0.796 & $(0.616-1.077)$ & 9.72 & $(5.07-12.80)$ & 8.99 & $(5.21-11.92)$ \\
\hline Fresh fruits & 2.009 & $(0.982-4.198)$ & 3.45 & $(1.97-6.43)$ & 1.87 & $(0.95-4.73)$ \\
\hline Dry fruits, nuts, and seeds & 11.131 & $(7.548-17.035)$ & 38.07 & $(16.64-89.51)$ & 4.48 & $(1.29-11.53)$ \\
\hline Sweets, chocolate, cakes, etc. & 0.696 & $(0.212-3.805)$ & 6.99 & $(3.32-69.53)$ & 7.08 & $(5.16-13.94)$ \\
\hline Oils and fats & 0.022 & $(0.000-0.123)$ & 0.43 & $(0.01-1.77)$ & 0.35 & $(0.004-1.48)$ \\
\hline \multirow[t]{2}{*}{ Beverages } & 0.671 & $(0.077-3.491)$ & 1.29 & $(0.52-3.06)$ & 2.36 & $(0.66-9.58)$ \\
\hline & 50 th & $\begin{array}{l}\text { Molybdenum }(\mu \mathrm{g} / \mathrm{kg}) \\
(\mathrm{IQR})\end{array}$ & 50 th & $\begin{array}{l}\text { Nickel }(\mu \mathrm{g} / \mathrm{kg}) \\
(\mathrm{IQR})\end{array}$ & 50 th & $\begin{array}{l}\text { Silver (ng/kg) } \\
(\mathrm{IQR})\end{array}$ \\
\hline Cereals and cereal products & 421.06 & (289.66-639.36) & 109.06 & $(67.03-183.42)$ & 374.85 & $(0.02-1026.00)$ \\
\hline Meat and meat products & 22.14 & $(12.70-41.98)$ & 27.07 & $(12.78-46.73)$ & 0.02 & $(0.02-234.00)$ \\
\hline Milk and dairy products & 77.10 & $(50.22-147.73)$ & 30.22 & $(16.27-95.61)$ & 0.02 & $(0.02-39.54)$ \\
\hline Eggs & 81.22 & (34.81-203.03) & 4.90 & $(3.30-7.21)$ & 0.02 & $(0.02-0.02)$ \\
\hline Fish and seafood & 9.82 & $(3.91-26.09)$ & 23.27 & $(13.06-50.22)$ & 376.00 & $(0.02-2319.00)$ \\
\hline All vegetables & 48.56 & $(20.29-101.79)$ & 64.75 & $(39.22-159.63)$ & 87.62 & $(0.02-550.00)$ \\
\hline Legumes & 2531.86 & $(1022.62-5399.85)$ & 896.13 & $(347.66-1660.21)$ & 3.00 & $(0.02-1778.00)$ \\
\hline Potatoes & 44.67 & $(40.48-199.30)$ & 97.14 & (75.89-194.37) & 282.00 & $(92.93-494.00)$ \\
\hline Fresh fruits & 9.19 & $(5.19-26.41)$ & 31.53 & $(13.39-59.45)$ & 0.02 & $(0.02-224.82)$ \\
\hline Dry fruits, nuts, and seeds & 163.32 & $(90.95-394.09)$ & 1096.59 & $(486.95-2087.37)$ & 734.00 & $(0.02-1881.00)$ \\
\hline Sweets, chocolate, cakes, etc. & 177.75 & $(79.66-244.11)$ & 98.45 & $(39.39-695.49)$ & 116.47 & $(0.02-537.32)$ \\
\hline Oils and fats & 1.87 & $(1.14-16.56)$ & 7.13 & $(2.67-16.82)$ & 0.02 & $(0.02-32.00)$ \\
\hline Beverages & 1.53 & $(0.70-3.76)$ & 14.31 & $(6.16-24.83)$ & 0.02 & $(0.02-0.15)$ \\
\hline
\end{tabular}


Table 2 (continued)

\begin{tabular}{|c|c|c|c|c|c|c|}
\hline & 50 th & $\begin{array}{l}\text { Strontium (mg/kg) } \\
\text { (IQR) }\end{array}$ & 50 th & $\begin{array}{l}\text { Tellurium }(\mu \mathrm{g} / \mathrm{kg}) \\
(\mathrm{IQR})\end{array}$ & 50 th & $\begin{array}{l}\text { Thallium }(\mu \mathrm{g} / \mathrm{kg}) \\
(\mathrm{IQR})\end{array}$ \\
\hline Cereals and cereal products & 1.262 & $(0.867-1.810)$ & 0.168 & $(0.000-1.022)$ & 0.055 & $(0.001-0.494)$ \\
\hline Meat and meat products & 0.222 & $(0.083-0.580)$ & 0.686 & $(0.292-1.605)$ & 0.052 & $(0.001-0.374)$ \\
\hline Milk and dairy products & 2.459 & $(0.851-3.975)$ & 0.937 & $(0.354-2.009)$ & 0.044 & $(0.001-0.210)$ \\
\hline Eggs & 0.317 & $(0.178-0.825)$ & 0.399 & $(0.142-0.482)$ & 0.442 & $(0.001-0.516)$ \\
\hline Fish and seafood & 1.286 & $(0.659-4.294)$ & 0.803 & $(0.283-1.631)$ & 0.006 & $(0.001-0.217)$ \\
\hline All vegetables & 1.846 & $(0.564-3.644)$ & 0.246 & $(0.000-0.674)$ & 0.256 & $(0.001-1.542)$ \\
\hline Legumes & 2.338 & $(1.477-3.669)$ & 0.382 & $(0.000-0.989)$ & 0.001 & $(0.001-0.343)$ \\
\hline Potatoes & 0.280 & $(0.208-0.630)$ & 0.189 & $(0.049-0.955)$ & 0.046 & $(0.001-0.509)$ \\
\hline Fresh fruits & 0.453 & $(0.197-1.722)$ & 0.185 & $(0.000-0.652)$ & 0.001 & $(0.001-0.134)$ \\
\hline Dry fruits, nuts, and seeds & 4.078 & $(1.797-7.432)$ & 1.072 & $(0.500-1.983)$ & 0.648 & $(0.054-2.002)$ \\
\hline Sweets, chocolate, cakes, etc. & 1.145 & $(0.472-4.363)$ & 0.435 & $(0.082-1.040)$ & 0.300 & $(0.031-1.478)$ \\
\hline Oils and fats & 0.017 & $(0.000-0.141)$ & 0.304 & $(0.000-1.451)$ & 0.001 & $(0.001-0.135)$ \\
\hline \multirow[t]{2}{*}{ Beverages } & 0.193 & $(0.046-0.580)$ & 0.048 & $(0.010-0.094)$ & 0.077 & $(0.020-0.230)$ \\
\hline & 50 th & $\begin{array}{l}\text { Titanium }(\mu \mathrm{g} / \mathrm{kg}) \\
(\mathrm{IQR})\end{array}$ & 50 th & $\begin{array}{l}\text { Uranium }(\mu \mathrm{g} / \mathrm{kg}) \\
(\mathrm{IQR})\end{array}$ & 50 th & $\begin{array}{l}\text { Vanadium }(\mu \mathrm{g} / \mathrm{kg}) \\
(\mathrm{IQR})\end{array}$ \\
\hline Cereals and cereal products & 395.84 & $(258.44-1109.75)$ & 0.470 & $(0.197-0.901)$ & 7.00 & $(3.28-12.92)$ \\
\hline Meat and meat products & 550.60 & $(333.29-2225.50)$ & 0.220 & $(0.068-0.429)$ & 3.46 & $(1.81-7.65)$ \\
\hline Milk and dairy products & 1100.75 & $(667.31-4800.02)$ & 0.340 & $(0.113-0.746)$ & 2.92 & $(1.59-7.44)$ \\
\hline Eggs & 450.00 & $(26.31-973.21)$ & 0.043 & $(0.039-0.178)$ & 1.41 & $(0.97-3.50)$ \\
\hline Fish and seafood & 453.96 & $(286.80-1342.46)$ & 0.790 & $(0.226-2.367)$ & 6.28 & $(3.05-17.67)$ \\
\hline All vegetables & 163.73 & $(65.93-471.55)$ & 0.185 & $(0.051-0.944)$ & 2.78 & $(1.12-9.12)$ \\
\hline Legumes & 790.47 & $(490.35-1031.42)$ & 0.301 & $(0.188-0.627)$ & 12.20 & $(3.22-26.60)$ \\
\hline Potatoes & 207.16 & $(85.09-447.81)$ & 0.133 & $(0.048-0.437)$ & 1.32 & $(0.97-2.37)$ \\
\hline Fresh fruits & 56.87 & $(30.32-152.52)$ & 0.051 & $(0.017-0.088)$ & 1.04 & $(0.59-1.72)$ \\
\hline Dry fruits, nuts, and seeds & 1222.86 & $(711.37-6671.81)$ & 0.170 & $(0.050-1.122)$ & 4.45 & $(1.91-9.80)$ \\
\hline Sweets, chocolate, cakes, etc. & 941.98 & $(414.39-1922.09)$ & 0.594 & $(0.262-1.383)$ & 8.62 & $(4.37-23.75)$ \\
\hline Oils and fats & 35.26 & $(10.87-111.97)$ & 0.003 & $(0.000-0.110)$ & 2.84 & $(1.07-3.79)$ \\
\hline Beverages & 76.67 & $(41.95-132.50)$ & 0.132 & $(0.050-0.304)$ & 1.90 & $(0.61-7.76)$ \\
\hline
\end{tabular}

results showing similar main sources of antimony content in food categories as well as our results show comparable (ANSES 2011; Arnich et al. 2012; Iyengar et al. 2000; Noel et al. 2003; Ysart et al. 1999) or slightly higher (Gibson and Scythes 1984; Gimou et al. 2014; Leblanc et al. 2005; Marcussen et al. 2013; Rose et al. 2010; Wappelhorst et al. 2002) or lower (Domingo et al. 2012) dietary intake respect to other European and non-European populations.

\section{Barium}

Barium compounds are present in nature due to leaching and erosion of natural deposits and subsequent contamination of groundwater sources. Foods showing higher barium content are cereals products, followed by legumes and dry fruits (Table 2). Particularly, among cereals, rice presents negligible content compared with pasta, bread, and other cereals products, while nuts and seeds, chocolate products, and other confectioneries show high concentration above $1 \mathrm{mg} / \mathrm{kg}$ (Supplemental Table S2). The estimated median dietary barium intake is $0.84 \mathrm{mg} /$ day (IQR $0.62-1.12 \mathrm{mg}$ / day) (Table 3 ) due to higher contribution from milk and dairy products (mainly aged cheese), cereal products, followed by vegetables (particularly leafy ones), and fruits (Supplemental Table S3). We found comparable intake (Gonzalez-Weller et al. 2013; Marcussen et al. 2013), or little higher than previous studies (ANSES 2011; Rose et al. 2010; Turconi et al. 2009; Ysart et al. 1999) and in one case lower (Gimou et al. 2014).

\section{Beryllium}

Main sources of beryllium and beryllium compounds are industrial processing and fossil fuel combustion (especially coal) resulting in emission of beryllium to the atmosphere, surface waters, and soil. In non-occupationally 
Table 3 Daily estimated trace element intake by food category

\begin{tabular}{|c|c|c|c|c|c|c|}
\hline & 50 th & $\begin{array}{l}\text { Antimony ( } \mu \mathrm{g} / \text { day) } \\
(\mathrm{IQR})\end{array}$ & 50th & $\begin{array}{l}\text { Barium ( } \mu \mathrm{g} / \text { day) } \\
(\mathrm{IQR})\end{array}$ & 50 th & $\begin{array}{l}\text { Beryllium (ng/day) } \\
\text { (IQR) }\end{array}$ \\
\hline Total intake ( $\mu \mathrm{g} /$ day $)$ & 3.471 & $(2.801-4.395)$ & 844.96 & $(618.13-1123.00)$ & 237.74 & $(179.06-319.22)$ \\
\hline Cereals and cereal products & 0.523 & $(0.303-0.765)$ & 177.52 & (117.78-259.18) & 37.98 & $(23.07-56.11)$ \\
\hline Meat and meat products & 0.496 & $(0.319-0.678)$ & 11.06 & $(7.44-17.50)$ & 8.94 & $(5.93-12.54)$ \\
\hline Milk and dairy products & 0.358 & $(0.213-0.579)$ & 251.11 & $(131.14-432.29)$ & 9.46 & $(5.36-13.95)$ \\
\hline Eggs & 0.010 & $(0.005-0.015)$ & 7.22 & $(3.90-11.34)$ & 0.37 & $(0.20-0.59)$ \\
\hline Fish and seafood & 0.081 & $(0.042-0.134)$ & 3.00 & $(1.57-5.03)$ & 4.42 & $(2.03-9.59)$ \\
\hline All vegetables & 0.396 & $(0.259-0.574)$ & 65.18 & $(42.06-100.70)$ & 49.78 & $(32.32-77.62)$ \\
\hline Legumes & 0.029 & $(0.013-0.054)$ & 18.89 & $(8.75-35.14)$ & 7.00 & $(3.24-13.03)$ \\
\hline Potatoes & 0.033 & $(0.020-0.060)$ & 2.78 & $(1.65-4.94)$ & 3.69 & $(2.19-6.56)$ \\
\hline Fresh fruits & 0.607 & $(0.387-0.870)$ & 78.55 & $(51.15-110.81)$ & 28.26 & $(17.81-40.82)$ \\
\hline Dry fruits, nuts, and seeds & 0.001 & $(0.001-0.006)$ & 13.38 & $(13.32-86.62)$ & 0.13 & $(0.08-0.69)$ \\
\hline Sweets, chocolate, cakes, etc. & 0.323 & $(0.179-0.581)$ & 44.42 & $(20.87-84.86)$ & 8.68 & $(3.92-15.22)$ \\
\hline Oils and fats & 0.162 & $(0.072-0.335)$ & 0.18 & $(0.13-0.25)$ & 0.47 & $(0.35-0.65)$ \\
\hline \multirow[t]{2}{*}{ Beverages } & 0.096 & $(0.042-0.191)$ & 28.46 & $(15.66-47.58)$ & 37.14 & $(7.66-103.82)$ \\
\hline & 50 th & $\begin{array}{l}\text { Boron }(\mu \mathrm{g} / \text { day }) \\
(\mathrm{IQR})\end{array}$ & 50 th & $\begin{array}{l}\text { Cobalt ( } \mu \mathrm{g} / \text { day) } \\
(\mathrm{IQR})\end{array}$ & 50 th & $\begin{array}{l}\text { Lithium ( } \mu \mathrm{g} / \text { day) } \\
(\mathrm{IQR})\end{array}$ \\
\hline Total intake ( $\mu \mathrm{g} /$ day $)$ & 2037.18 & $(1511.17-2760.72)$ & 19.680 & $(14.822-25.170)$ & 18.151 & (14.638-22.868) \\
\hline Cereals and cereal products & 112.99 & (76.37-156.77) & 1.729 & $(1.122-2.527)$ & 3.651 & $(2.445-5.289)$ \\
\hline Meat and meat products & 40.56 & $(27.15-56.36)$ & 0.516 & $(0.334-0.730)$ & 0.750 & $(0.496-1.071)$ \\
\hline Milk and dairy products & 45.18 & $(25.16-70.42)$ & 1.115 & $(0.694-1.735)$ & 1.079 & $(0.617-1.605)$ \\
\hline Eggs & 3.44 & $(1.86-5.41)$ & 0.043 & $(0.023-0.068)$ & 0.087 & $(0.047-0.137)$ \\
\hline Fish and seafood & 11.55 & $(5.56-19.57)$ & 0.271 & $(0.129-0.537)$ & 0.792 & $(0.382-1.475)$ \\
\hline All vegetables & 238.99 & $(162.30-344.08)$ & 5.889 & $(3.593-9.684)$ & 4.912 & $(3.263-6.951)$ \\
\hline Legumes & 115.51 & $(53.51-214.89)$ & 1.253 & $(0.580-2.330)$ & 0.669 & $(0.310-1.245)$ \\
\hline Potatoes & 19.44 & $(11.56-34.56)$ & 0.285 & $(0.169-0.506)$ & 0.164 & $(0.097-0.291)$ \\
\hline Fresh fruits & 676.60 & (428.84-973.63) & 1.437 & $(0.918-2.053)$ & 1.723 & $(1.098-2.456)$ \\
\hline Dry fruits, nuts, and seeds & 3.70 & $(2.75-20.20)$ & 0.022 & $(0.018-0.130)$ & 0.033 & $(0.006-0.091)$ \\
\hline Sweets, chocolate, cakes, etc. & 86.66 & (40.56-149.54) & 1.832 & $(0.913-3.534)$ & 0.714 & $(0.398-1.192)$ \\
\hline Oils and fats & 4.36 & $(3.19-6.00)$ & 0.018 & $(0.012-0.026)$ & 0.008 & $(0.004-0.014)$ \\
\hline \multirow[t]{2}{*}{ Beverages } & 326.13 & $(83.81-934.67)$ & 1.806 & $(1.002-3.336)$ & 1.677 & $(0.715-3.330)$ \\
\hline & 50 th & $\begin{array}{l}\text { Molybdenum ( } \mu \mathrm{g} / \text { day) } \\
(\mathrm{IQR})\end{array}$ & 50 th & $\begin{array}{l}\text { Nickel ( } \mu \mathrm{g} / \text { day) } \\
(\mathrm{IQR})\end{array}$ & 50 th & $\begin{array}{l}\text { Silver (ng/day) } \\
\text { (IQR) }\end{array}$ \\
\hline Total intake ( $\mu \mathrm{g} /$ day $)$ & 196.28 & (150.31-260.36) & 130.92 & $(102.80-168.94)$ & 908.14 & $(679.09-1146.63)$ \\
\hline Cereals and cereal products & 74.36 & $(50.38-104.76)$ & 24.53 & (16.04-34.99) & 226.10 & $(140.84-325.60)$ \\
\hline Meat and meat products & 4.07 & $(2.56-6.06)$ & 3.92 & $(2.55-5.34)$ & 43.70 & $(23.87-87.08)$ \\
\hline Milk and dairy products & 12.70 & $(7.31-18.71)$ & 8.03 & $(4.49-11.75)$ & 11.41 & $(6.55-18.98)$ \\
\hline Eggs & 2.15 & $(1.16-3.38)$ & 0.08 & $(0.04-0.12)$ & 0.01 & $(0.01-0.01)$ \\
\hline Fish and seafood & 0.58 & $(0.28-1.03)$ & 2.10 & $(1.15-3.57)$ & 86.65 & $(39.07-208.52)$ \\
\hline All vegetables & 12.91 & $(8.73-18.87)$ & 13.62 & $(8.94-20.21)$ & 73.95 & $(48.68-113.36)$ \\
\hline Legumes & 51.99 & $(24.09-96.72)$ & 16.61 & $(7.70-30.90)$ & 18.18 & $(8.42-33.82)$ \\
\hline Potatoes & 2.48 & $(1.48-4.41)$ & 2.50 & $(1.49-4.45)$ & 8.00 & $(4.76-14.22)$ \\
\hline Fresh fruits & 5.91 & $(3.77-8.49)$ & 14.15 & $(9.04-19.96)$ & 173.03 & (107.74-251.37) \\
\hline Dry fruits, nuts, and seeds & 0.18 & $(0.17-1.17)$ & 0.43 & $(0.41-2.69)$ & 1.37 & $(0.53-5.09)$ \\
\hline Sweets, chocolate, cakes, etc. & 9.31 & $(3.44-15.99)$ & 13.82 & $(5.64-30.39)$ & 36.23 & $(16.52-72.89)$ \\
\hline Oils and fats & 0.30 & $(0.16-0.58)$ & 1.23 & $(0.91-1.68)$ & 38.13 & $(12.26-85.62)$ \\
\hline Beverages & 1.78 & $(0.96-3.39)$ & 9.29 & $(5.36-15.32)$ & 3.04 & $(2.00-4.67)$ \\
\hline
\end{tabular}


Table 3 (continued)

\begin{tabular}{|c|c|c|c|c|c|c|}
\hline & 50th & $\begin{array}{l}\text { Strontium ( } \mu \mathrm{g} / \text { day) } \\
(\mathrm{IQR})\end{array}$ & 50 th & $\begin{array}{l}\text { Tellurium (ng/day) } \\
\text { (IQR) }\end{array}$ & 50 th & $\begin{array}{l}\text { Thallium (ng/day) } \\
\text { (IQR) }\end{array}$ \\
\hline Total intake ( $\mu \mathrm{g} /$ day $)$ & 1931.99 & $(1543.32-2390.35)$ & 2698.06 & $(1921.86-3752.08)$ & 533.69 & $(414.58-676.04)$ \\
\hline Cereals and cereal products & 248.66 & $(162.64-361.39)$ & 177.77 & $(111.81-255.98)$ & 65.87 & (39.14-99.96) \\
\hline Meat and meat products & 43.72 & $(28.49-65.50)$ & 339.40 & (217.28-499.84) & 73.64 & $(46.41-109.50)$ \\
\hline Milk and dairy products & 212.45 & $(132.59-323.31)$ & 1089.56 & $(605.00-1863.86)$ & 28.58 & $(12.89-44.78)$ \\
\hline Eggs & 6.38 & $(3.45-10.02)$ & 6.91 & $(3.73-10.84)$ & 6.53 & $(3.53-10.25)$ \\
\hline Fish and seafood & 70.68 & $(34.05-129.31)$ & 36.17 & $(18.63-64.91)$ & 4.83 & $(2.03-9.87)$ \\
\hline All vegetables & 405.23 & $(259.54-612.72)$ & 106.63 & $(72.36-151.16)$ & 123.22 & $(67.33-200.73)$ \\
\hline Legumes & 37.51 & $(17.38-69.78)$ & 9.03 & $(4.18-16.80)$ & 4.92 & $(2.28-9.16)$ \\
\hline Potatoes & 9.70 & $(5.77-17.25)$ & 11.01 & $(6.55-19.58)$ & 9.49 & $(5.64-16.87)$ \\
\hline Fresh fruits & 400.95 & $(251.58-562.46)$ & 245.29 & $(155.58-354.65)$ & 51.86 & $(33.03-73.21)$ \\
\hline Dry fruits, nuts, and seeds & 3.13 & $(2.65-19.03)$ & 0.54 & $(0.42-3.09)$ & 1.13 & $(0.63-5.70)$ \\
\hline Sweets, chocolate, cakes, etc. & 93.08 & $(46.63-159.51)$ & 48.00 & $(27.39-76.21)$ & 42.75 & $(20.27-77.95)$ \\
\hline Oils and fats & 1.31 & $(0.67-2.39)$ & 309.04 & $(123.69-626.20)$ & 0.56 & $(0.38-0.86)$ \\
\hline \multirow[t]{2}{*}{ Beverages } & 155.96 & $(77.35-292.84)$ & 42.38 & $(26.89-61.84)$ & 48.25 & $(23.53-87.42)$ \\
\hline & 50 th & $\begin{array}{l}\text { Titanium ( } \mu \mathrm{g} / \text { day) } \\
(\mathrm{IQR})\end{array}$ & 50 th & $\begin{array}{l}\text { Uranium (ng/day) } \\
\text { (IQR) }\end{array}$ & 50 th & $\begin{array}{l}\text { Vanadium ( } \mu \mathrm{g} / \text { day) } \\
(\mathrm{IQR})\end{array}$ \\
\hline Total intake ( $\mu \mathrm{g} /$ day $)$ & 881.90 & $(705.58-1135.53)$ & 790.18 & $(600.52-1121.22)$ & 10.363 & $(7.822-13.426)$ \\
\hline Cereals and cereal products & 140.37 & (93.06-198.58) & 154.95 & $(98.57-224.00)$ & 1.646 & $(1.010-2.463)$ \\
\hline Meat and meat products & 155.74 & $(107.32-219.56)$ & 31.67 & $(20.58-44.52)$ & 0.645 & $(0.425-0.925)$ \\
\hline Milk and dairy products & 283.99 & $(167.35-407.48)$ & 59.46 & $(35.03-96.26)$ & 0.370 & $(0.229-0.528)$ \\
\hline Eggs & 7.64 & $(4.13-12.00)$ & 3.30 & $(1.79-5.19)$ & 0.032 & $(0.017-0.050)$ \\
\hline Fish and seafood & 33.13 & $(18.73-52.28)$ & 48.97 & $(20.37-116.35)$ & 0.560 & $(0.270-1.081)$ \\
\hline All vegetables & 51.81 & $(36.36-77.02)$ & 117.53 & $(77.26-175.57)$ & 2.025 & $(1.330-3.131)$ \\
\hline Legumes & 11.02 & $(5.10-20.49)$ & 7.31 & $(3.39-13.61)$ & 0.255 & $(0.118-0.474)$ \\
\hline Potatoes & 4.94 & $(2.94-8.78)$ & 6.57 & $(3.91-11.68)$ & 0.188 & $(0.112-0.335)$ \\
\hline Fresh fruits & 32.05 & $(20.45-44.86)$ & 19.51 & $(12.29-28.19)$ & 0.355 & $(0.226-0.510)$ \\
\hline Dry fruits, nuts, and seeds & 0.91 & $(0.80-5.63)$ & 0.46 & $(0.25-2.23)$ & 0.005 & $(0.002-0.015)$ \\
\hline Sweets, chocolate, cakes, etc. & 57.30 & (29.02-108.07) & 71.74 & $(39.63-128.74)$ & 0.860 & $(0.474-1.515)$ \\
\hline Oils and fats & 5.19 & $(0.00-19.75)$ & 1.33 & $(0.83-2.25)$ & 0.064 & $(0.042-0.097)$ \\
\hline Beverages & 2.23 & $(1.34-3.57)$ & 135.17 & $(53.23-332.62)$ & 1.516 & $(0.479-3.681)$ \\
\hline
\end{tabular}

exposed population, ingestion through foods and beverages is the main route of exposure (WHO 2009). We found higher beryllium concentration in legumes, dry fruits, and sweet products (Table 2), with also substantial content in all cereal products but rice, leafy vegetables, red wine, and chocolate and biscuits/dry cakes (Supplemental Table S2). Beryllium daily intake is of $0.24 \mu \mathrm{g} /$ day (IQR 0.18-0.32 $\mu \mathrm{g} /$ day) (Table 3) with main contribution from leafy vegetables, cereals (pasta and bread), beverages (particularly wine), and citrus fruits (Supplemental Table S3). We found comparable intake (ATSDR 2007; Domingo et al. 2012; Marcussen et al. 2013) or in such cases little higher (Llobet et al. 1998; Turconi et al. 2009) than previous studies.

\section{Boron}

The collective body of evidence has yet to establish a clear biological function for boron in humans, since no specific biochemical function has been identified (Institute of Medicine 2001). Adverse health effects due to low boron intake have been consistently accompanied with deficiency of other elements such as calcium, copper, or magnesium (Nielsen 2014; WHO 2003d). The greatest amount of boron exposure in the general population occurs through food intake in the form of borate and boric acid (European Food Safety Authority 2004c). We found higher boron concentration in dry fruits/seeds, legumes, and fruits (Table 2), with also high content among all vegetables from leafy and other vegetables, cabbage, chocolate and candy bars, and wine 
Table 4 Dietary daily intake (DI) and tolerable upper intake levels (UI) of trace elements reported by this study compared with data reported by international agencies

\begin{tabular}{|c|c|c|c|c|c|c|c|}
\hline \multirow[t]{2}{*}{ Element } & \multirow[b]{2}{*}{ DI ( $\mu$ g or mg/day) } & \multicolumn{2}{|l|}{ This study } & \multirow{2}{*}{$\begin{array}{l}\text { EU } \\
\mathrm{UI}(\mu \mathrm{g} \text { or } \mathrm{mg} / \mathrm{kg} \mathrm{bw} / \\
\text { week) }\end{array}$} & \multirow{2}{*}{$\begin{array}{l}\text { WHO } \\
\text { UI ( } \mu \text { g or mg/kg bw/ } \\
\text { week }\end{array}$} & \multirow{2}{*}{$\begin{array}{l}\text { US-EPA } \\
\mathrm{RfD}^{\mathrm{a}}(\mu \mathrm{g} \text { or } \\
\mathrm{mg} / \mathrm{kg} \text { bw/ } \\
\text { day) }\end{array}$} & \multirow[t]{2}{*}{ THQ } \\
\hline & & $\begin{array}{l}\mathrm{DI}(\mu \mathrm{g} \text { or } \\
\mathrm{mg} / \mathrm{kg} \mathrm{bw} / \\
\text { day) }\end{array}$ & $\begin{array}{l}\mathrm{DI}(\mu \mathrm{g} \text { or } \\
\mathrm{mg} / \mathrm{kg} \text { bw/ } \\
\text { week) }\end{array}$ & & & & \\
\hline Antimony $(\mathrm{Sb})(\mu \mathrm{g})$ & 3.47 & 0.050 & 0.352 & $\begin{array}{l}6 \text { (European Food } \\
\text { Safety Authority } \\
\text { 2004b) }\end{array}$ & 6 (WHO 2003b) & 4 & 0.013 \\
\hline Barium (Ba) (mg) & 0.84 & 0.012 & 0.084 & 0.2 (SCHER 2012) & 0.21 (WHO 2016) & 0.2 & 0.060 \\
\hline Beryllium (Be) ( $\mu \mathrm{g})$ & 0.24 & 0.003 & 0.024 & Not assessed & $\begin{array}{l}2 \text { (Bruce et al. 2001; } \\
\text { WHO 2009) }\end{array}$ & 3 & 0.001 \\
\hline Boron (B) (mg) & 2.05 & 0.029 & 0.206 & $\begin{array}{l}0.14 \text { (European Food } \\
\text { Safety Authority } \\
2004 c)^{b}\end{array}$ & $0.19{(\text { WHO } 1996)^{b}}^{b}$ & 0.2 & 0.150 \\
\hline Cobalt $(\mathrm{Co})(\mu \mathrm{g})$ & 19.68 & 0.277 & 1.938 & $\begin{array}{l}\text { 1.6 (European Food } \\
\text { Safety Authority } \\
\text { 2012) }\end{array}$ & $\begin{array}{l}\text { Not derived (Kim } \\
\text { et al. 2006) }\end{array}$ & $5^{c}$ & $0.560^{\mathrm{e}}$ \\
\hline Lithium $(\mathrm{Li})(\mu \mathrm{g})$ & 18.15 & 0.258 & 1.807 & $\begin{array}{l}\text { Not derived (European } \\
\text { Food Safety Author- } \\
\text { ity 2009a) }\end{array}$ & $\begin{array}{l}\text { Not derived (WHO } \\
\text { 1996) }\end{array}$ & $2^{d}$ & 0.130 \\
\hline $\begin{array}{l}\text { Molybdenum (Mo) } \\
\quad(\mathrm{mg})\end{array}$ & 0.20 & 0.003 & 0.020 & $\begin{array}{l}0.009 \text { (European Food } \\
\text { Safety Authority } \\
2013)^{\mathrm{b}}\end{array}$ & $\begin{array}{l}0.029 \text { (Institute of } \\
\text { Medicine 2001; } \\
\text { WHO 2003a) }\end{array}$ & 0.005 & 0.560 \\
\hline Nickel (Ni) ( $\mu \mathrm{g})$ & 130.92 & 1.844 & 12.906 & $\begin{array}{l}2.8 \text { (European Food } \\
\text { Safety Authority } \\
\text { 2015) }\end{array}$ & 22 (WHO 2005) & 20 & 0.092 \\
\hline Silver $(\mathrm{Ag})(\mu \mathrm{g})$ & 0.91 & 0.013 & 0.089 & $\begin{array}{l}\text { Not derived (European } \\
\text { Food Safety Author- } \\
\text { ity 2016b) }\end{array}$ & $\begin{array}{l}\text { Not derived (WHO } \\
\text { 2003c) }\end{array}$ & 5 & 0.003 \\
\hline Strontium (Sr) (mg) & 1.93 & 0.027 & 0.191 & $\begin{array}{l}\text { Not derived (European } \\
\text { Food Safety Author- } \\
\text { ity 2009c) }\end{array}$ & $0.13($ WHO 2010) & 0.6 & 0.045 \\
\hline Tellurium $(\mathrm{Te})(\mu \mathrm{g})$ & 2.70 & 0.039 & 0.271 & $\begin{array}{l}\text { Not derived (ANSES } \\
\text { 2011) }\end{array}$ & Not assessed & 210 & 0.0002 \\
\hline Thallium $(\mathrm{Tl})(\mu \mathrm{g})$ & 0.53 & 0.007 & 0.052 & $\begin{array}{l}0.14 \text { (German Federal } \\
\text { Institute for Risk } \\
\text { Assessment (BfR) } \\
2004)^{\mathrm{b}}\end{array}$ & $\begin{array}{l}\text { Not derived (WHO } \\
\text { and International } \\
\text { Programme for } \\
\text { Chemical Safety } \\
\text { 1996) }\end{array}$ & $0.01^{\mathrm{e}}$ & 0.744 \\
\hline Titanium (Ti) (mg) & 0.88 & 0.126 & 0.885 & $\begin{array}{l}\text { Not derived (European } \\
\text { Food Safety Authority } \\
\text { 2016a; European Food } \\
\text { Safety Authority 2018; } \\
\text { European Food Safety } \\
\text { Authority 2019) }\end{array}$ & $\begin{array}{l}\text { Not derived (FAO/ } \\
\text { WHO 1969) }\end{array}$ & Not assessed & - \\
\hline Uranium $(\mathrm{U})(\mu \mathrm{g})$ & 0.79 & 0.012 & 0.081 & $\begin{array}{l}0.6 \text { (European Food } \\
\text { Safety Authority } \\
\text { 2009b) }\end{array}$ & 0.6 (WHO 2004) & 3 & 0.004 \\
\hline Vanadium $(V)(\mu \mathrm{g})$ & 10.36 & 0.146 & 1.021 & $\begin{array}{l}\text { Not derived (European } \\
\text { Food Safety Authority } \\
\text { 2004a; Tiesjema and } \\
\text { Baars 2009) }\end{array}$ & $\begin{array}{l}\text { Not derived (Institute } \\
\text { of Medicine 2001; } \\
\text { WHO 1996; WHO } \\
\text { 2000) }\end{array}$ & 9 & 0.017 \\
\hline
\end{tabular}

Data for target hazard quotient (THQ) calculation reported in bold

$b w$ body weight, $D I$ estimated dietary intake, EFSA European Food Safety Authority, UI upper intake daily intake, US-EPA US-Environmental Protection Agency, RfD reference dose, $T H Q$ target hazard quotient, WHO World Health Organization

${ }^{a}$ Reference dose (RfD) provided by US-Environmental Protection Agency (US-EPA) (US-EPA) 
Table 4 (continued)

${ }^{\mathrm{b}} 70 \mathrm{~kg}$ of body weight considered for the comparison. Original values for boron were $10 \mathrm{mg} /$ day (European Food Safety Authority $2004 \mathrm{c}$ ) and $13 \mathrm{mg} /$ day (WHO 1996), for molybdenum $0.6 \mathrm{mg} /$ day (European Food Safety Authority 2013) and $2 \mathrm{mg} / \mathrm{day}$ (Institute of Medicine 2001; WHO 2003a) and for thallium $10 \mathrm{mg} /$ day (German Federal Institute for Risk Assessment (BfR) 2004)

${ }^{c}$ Using the subchronic p-RfD of $3 \mathrm{mg} / \mathrm{kg} /$ day, the THQ is 0.093 (Finley et al. 2012)

${ }^{\mathrm{d}}$ For lithium, we used the subchronic and chronic p-RfD of $2 \mu \mathrm{g} / \mathrm{kg}$-day since RfD is not available

${ }^{\mathrm{e}}$ Since RfD was not derived, we used the value of $0.01 \mu \mathrm{g} / \mathrm{kg}$ bw/day for thallium salts, observed for hair follicle atrophy

${ }^{\mathrm{f}}$ Upper limit not derived for elemental titanium, but for titanium dioxide $\left(\mathrm{TiO}_{2}\right)$ a No Observed Adverse Effect Level (NOAEL) of $2250 \mathrm{mg}$ $\mathrm{TiO} 2 / \mathrm{kg}$ bw/day was reported

${ }^{\mathrm{g}}$ Upper limit not derived, although the lowest intake with reported adverse effects was $200 \mu \mathrm{g} / \mathrm{kg}$ bw/day. The Institute of Medicine proposed a values of $1.8 \mathrm{mg} /$ day as upper limit for elemental vanadium (Institute of Medicine 2001), approximately corresponding to $0.18 \mathrm{mg} / \mathrm{kg} \mathrm{bw} / \mathrm{week}$ for an adult of $70 \mathrm{~kg}$ of body weight

(Supplemental Table S2). Estimated dietary boron intake is $2.05 \mathrm{mg} /$ day (IQR $1.51-2-76 \mathrm{mg} /$ day) that is comparable or little higher compared to the data reported in previous studies (Biego et al. 1998; Hunt and Meacham 2001; Iyengar et al. 2000; Meacham and Hunt 1998; Rainey and Nyquist 1998; Shimbo et al. 1996; Turconi et al. 2009; Ysart et al. 1999).

\section{Cobalt}

Cobalt owes its essentiality as central bonding atom for the vitamin B12 (also called cobalamin), which is necessary for the metabolism of folates and fatty acids. In the general population, the largest source of cobalt exposure is through diet, ingested in its inorganic form by plants, while only a small fraction of total cobalt intake occurs in the form of cobalamin from foods of animal origin (Gambelli et al. 1999; Kim et al. 2006). In our study, foods showing the highest cobalt content are legumes, dry fruits/seeds, and potatoes (Table 2), with also high levels in sweets (particularly chocolate products), leafy vegetables, offal, and cheese (Supplemental Table S2). The daily cobalt intake is $19 \mu \mathrm{g} / \mathrm{day}$ (IQR 14.82-25.17 $\mu \mathrm{g} /$ day), driven by vegetables, with also substantial contribution from sweets, beverages, cereals, but also fresh fruits, legumes, and dairy products. Dietary intake was similar (Domingo et al. 2012; Reilly 2002) or slightly higher than other populations (ANSES 2011; Arnich et al. 2012; Marcussen et al. 2013; Ysart et al. 1999), but alternatively higher (Leblanc et al. 2005; Noel et al. 2003; Shimbo et al. 1996) and lower intake (Biego et al. 1998; Turconi et al. 2009) are also reported.

\section{Lithium}

Lithium is the lightest alkali metal, naturally present in soil and water. Due to its similarity to sodium and potassium and to a lesser extent to magnesium and calcium, it can compete with their intracellular targets and binding sites, although with different affinity (WHO 1996). In spite of lithium not yet considered an essential element
(Schrauzer 2002), it has been demonstrated that lithium plays a role within the nervous system, and lithium salts are used in the treatment of psychiatric diseases, especially bipolar affective disorder (Mitchell and HadziPavlovic 2000). According to main categories, we found the highest lithium content in fish (especially crustaceans and molluscs), legumes, cereal products (all but rice), and potatoes (Table 2), with also high concentration in dry fruits, leafy vegetables and cabbage, sweet confectionery not chocolate, red wine, and fresh cheese (Supplemental Table S2). Our results show an estimated dietary lithium intake of $18.15 \mu \mathrm{g} /$ day (IQR 7.16-29.15 $\mu \mathrm{g} /$ day) comparable or slightly higher to previous studies (Evans et al. 1985; Leblanc et al. 2005; Marcussen et al. 2013; Noel et al. 2003; Ysart et al. 1999), though also higher intake was reported (Gimou et al. 2014; Gonzalez-Weller et al. 2013; Iyengar et al. 2000; Turconi et al. 2009).

\section{Molybdenum}

Molybdenum is considered an essential trace element as it enters in a cofactor (molybdopterin) of certain enzymes that catalyse redox reactions (European Food Safety Authority 2013). Legumes, cereals (particularly rice), and offal are foods generally containing high concentration of molybdenum, similarly to what we found in our study (Table 2), and also chocolate, biscuits, dry cakes, and dry fruits/seeds present high content (Supplemental Table S2). Dietary daily intake is $196.28 \mu \mathrm{g} /$ day (IQR 150.31-260.36 $\mu \mathrm{g} /$ day) due to substantial contribution from legumes, cereals (Table 3), particularly pasta and bread, but also vegetables, milk, and dairy products (Supplemental Table S3). Our estimates are similar (Gimou et al. 2014; Hunt and Meacham 2001; Leblanc et al. 2005; Shimbo et al. 1996) or slightly higher compared to other studies (ANSES 2011; Evans et al. 1985; Noel et al. 2003; Turconi et al. 2009; Ysart et al. 1999), while lower than other findings (Biego et al. 1998; Marcussen et al. 2013). 


\section{Nickel}

Nickel is not essential for humans despite it plays an essential role in methionine metabolism in other animal species (WHO 2005). Conversely, nickel compounds are considered human carcinogens following exposure by inhalation (IARC 1990). However, there are no studies indicating any carcinogenic effects following oral exposure (IARC 1990). Beside raw foods, nickel levels in processed foods can be increased by pick-up from cooking materials and containers (Reilly 2002). Foods showing high nickel concentrations are mainly dry fruits/seeds and legumes (Table 2), with also very high levels in chocolate products, and partially also mushrooms, rice, and bread (Supplemental Table S2). The main foods contributing to the nickel daily intake of 130.92 $\mu \mathrm{g} /$ day (IQR 102.80-168-94 $\mu \mathrm{g}$ / day) are cereals, legumes, vegetables, fresh fruits, and sweets (Table 3), particularly leafy vegetables, other vegetables and tomatoes, citrus fruits, chocolate products, and coffee and tea (Supplemental Table S3). Similar intake is reported in previous studies (Alberti-Fidanza et al. 2002; Bocio et al. 2005; Pennington and Jones 1987; Shimbo et al. 1996; Ysart et al. 1999), while little lower (ANSES 2011; Arnich et al. 2012; Gimou et al. 2014; Larsen et al. 2002; Marcussen et al. 2013; Turconi et al. 2009), and alternatively higher was reported in other ones (Domingo et al. 2012; Leblanc et al. 2005; Santos et al. 2004).

\section{Silver}

Silver is a white metal generally having only trace amounts in foods, apart from its possible use as food additive (i.e. colouring agent) in cake decorations and confectionery (European Food Safety Authority 2016b). We found higher silver concentration in dry fruits, nuts, and seeds, followed by fish and seafood, cereals products, and eventually potatoes and sweets (Table 2). In particular, we found the highest values in crustaceans and molluscs, mushrooms, rice, and dry fruits. The estimated daily dietary intake is $0.908 \mu \mathrm{g} /$ day (IQR $0.679-1.147 \mu \mathrm{g} /$ day) with main contribution from cereals, fruits, and vegetables (mainly leafy ones), and fish, particularly crustaceans and molluscs (Table 3 and Supplemental Table S3). It should be pointed out that approximately half of the samples showed values below the LOD for silver (Supplemental Table S1), thus estimated could have been influenced by this large number of low results. Though limited dietary intake data are reported for silver (European Food Safety Authority 2011; Reilly 2002), our results show lower intake compared with other populations (ANSES 2011; Arnich et al. 2012; Dolara 2014; Evans et al. 1985; Gibson and Scythes 1984; Marcussen et al. 2013).

\section{Strontium}

Strontium occurs naturally in Earth's crust in the form of minerals such as celestite and strontianite and for humans non-occupationally exposed major sources are drinking water and foods (WHO 2010). Strontium can interfere with bone mineralization in the developing skeleton (WHO 2010), and a relationship between strontium exposure and childhood rickets has been suggested (Ozgur et al. 1996). We found the greater strontium content in dry fruits/seeds, dairy products, and legumes (Table 2) with the highest levels in crustaceans and molluscs, sweets products like confectionery made with and without chocolate, aged cheese, and some types of vegetables, particularly leafy, root, cabbage, and other types (Supplemental Table S2). The estimated dietary strontium intake was of $1.93 \mathrm{mg} /$ day (IQR $1.54-2.39 \mathrm{mg}$ / day), mainly driven by vegetables and fruits, followed by cereals (mainly bread and pasta) and dairy products (particularly cheese) (Table 3 and Supplemental Table S3). We found generally comparable (ANSES 2011; Gimou et al. 2014; Gonzalez-Weller et al. 2013; Iyengar et al. 2000; WHO 2010) or slightly higher estimated intake than previous studies (Evans et al. 1985; Marcussen et al. 2013; Turconi et al. 2009; Ysart et al. 1999).

\section{Tellurium}

Tellurium is a rare trace element with no biological function in human and generally its importance as a food contaminant is minor (Reilly 2002), as confirmed in our study demonstrating approximately $20 \%$ of samples below the LOD (Supplemental Table S1). In particular, we found higher tellurium concentrations in dry fruits, nuts and seeds, milk and dairy products, and fish (Table 2). Particularly in subgroup categories, we found the highest tellurium levels in processed meat, aged cheese, and mushrooms (Supplemental Table S2). The estimated daily dietary intake is $2.70 \mu \mathrm{g} /$ day (IQR 1.92-3.75 $\mu \mathrm{g} /$ day) driven by foods of animal origin, namely dairy products, red and processed meat, oils and fats, followed by citrus fruits and cereals (Table 3 and Supplemental Table S3). Despite the limited data available (Reilly 2002), we found consistent findings with the most recent studies (ANSES 2011; Gimou et al. 2014), demonstrating a much lower intake than older data previously reported (Kron et al. 1991; Reilly 2002; Schroeder et al. 1967).

\section{Thallium}

Little information is available on levels of thallium in foods and diets. There is some evidence that thallium behaves like potassium in soil, thus it is readily absorbed by certain plants (Reilly 2002). In our study, we observed higher thallium concentration in dry fruits/seeds, vegetables, and eggs 
(Table 2), with the highest levels in root vegetables, cabbages, and particularly chocolate products (Supplemental Table S2). Thallium dietary intake was estimated in $0.53 \mu \mathrm{g} /$ day (IQR 0.41-0.68 $\mu \mathrm{g} /$ day), with main contribution from vegetables (mainly cabbages, root, and other vegetables), followed by meat (primarily white), cereal products (all but rice), and fresh fruits, particularly citrus ones (Table 3 and Supplemental Table S3). Also for thallium, we observed a consistent number (35.8\%) of samples below the LOD (Supplemental Table S1), and also limited data about intake are available in other population. Nevertheless, our findings suggest that a lower dietary intake seems to be experienced compared with previous studies (Domingo et al. 2012; Rose et al. 2010; Y sart et al. 1999).

\section{Titanium}

Titanium is the eighth most common element in Earth's crust and is commonly found in foods. In addition, in food industry, titanium dioxide is used as food additive for whitening and brightening purpose in flour, confection, and other sweets products, and non-dairy milk products (European Food Safety Authority 2016a; Reilly 2002) as well as in other personal care products, e.g. toothpaste, cosmetics, or sunscreens (Rompelberg et al. 2016; Weir et al. 2012; Winkler et al. 2018). Due to its wide-scale distribution in the environment, titanium is a frequent food contaminant, but at relatively low levels since it is poorly absorbed from soil (Reilly 2002). We found high metal concentrations in dry fruits/seeds, dairy products (particularly cheese), sweets, and also legumes (Table 2). In particular, we detected the highest titanium level in chocolate products and biscuits/dry cakes among sweets, and also white meat compared with overall content (Supplemental Table S2). We estimated a dietary intake of $0.88 \mathrm{mg} /$ day (IQR $0.71-1.14 \mathrm{mg} /$ day), with major contribution from milk and dietary products, meat, and cereals. In spite of its wide use, data on dietary intake of total titanium are scarce and not up-to-date. Our results showed more than double intake compared with a previous study carried out in Japan and US (Reilly 2002; Shimbo et al. 1996). Conversely, due to the large use in the most recent decades of titanium dioxide, many studies assessed its particular intake, showing intake levels approximately similar or slightly higher than our study (Bachler et al. 2015; Heringa et al. 2016; Rompelberg et al. 2016; Weir et al. 2012; Winkler et al. 2018), also considering that most of them accounted the contribution from other sources like toothpaste that could not be considered in our study.

\section{Uranium}

Uranium exposure in the general population is only marginally assessed. Excluding contamination from dumping sites, uranium is naturally present in soil at different levels depending on the geological origin, thus affecting the natural content in waters and foods and consequently human dietary intake (Anke et al. 2009; European Food Safety Authority 2009b). We found high uranium levels in fish, sweets products, and cereals (Table 2), with the highest levels in crustaceans and molluscs, mushrooms, dry fruits, and also leafy vegetables (Supplemental Table S2). We estimated a daily intake of $0.79 \mu \mathrm{g} /$ day (IQR $0.60-1.12 \mu \mathrm{g}$ / day) due to contribution by cereals (all but rice), beverages (particularly fruit juices, coffee/tea, and wine), and vegetables (leafy and other vegetables and tomatoes) (Table 3 and Supplemental Table S3). We found comparable intake compared with one previous study (Marcussen et al. 2013) while notably lower than those found in one Spanish survey (Domingo et al. 2012).

\section{Vanadium}

In spite of some indication for symptoms of deficiency in animals, vanadium has not been shown to be essential for humans and thus it has no nutritional value (European Food Safety Authority 2004a). Nevertheless, in the general population diet is the major source of the metal (Institute of Medicine 2001). Our findings show that legumes and sweets, cereals, and also fish are foods showing high vanadium content (Table 2), with the highest values in chocolate products, dry fruits, leafy vegetables, crustaceans and molluscs, and bread (Supplemental Table S2). The estimated daily intake is $10.36 \mu \mathrm{g} /$ day (IQR 7.82-13.43 $\mu \mathrm{g}$ / day), due to major contribution from vegetables, cereals, and beverages (Table 3), particularly leafy vegetables, bread, and wine from subgroup analysis (Supplemental Table S3). Similar or slightly lower intake was found compared to previous studies (Arnich et al. 2012; Evans et al. 1985; Gimou et al. 2014; Pennington and Jones 1987; Shimbo et al. 1996; Turconi et al. 2009), except when comparing with findings from two Spanish studies reporting much higher intake (Bocio et al. 2005; Domingo et al. 2012).

\section{Risk Assessment}

For the considered elements, the target hazard quotient (THQ) shows no substantial risk when comparing the median intake in the study population with the RfD provided by US-EPA (Table 4). It should be noted that for titanium we are unable to evaluate the THQ since RfD was not provided, as well as no upper limits are derived from international agencies, considering the use of titanium substantially safe (European Food Safety Authority 2019; FAO/WHO 1969). 


\section{Conclusions}

Overall, our study provides dietary estimated intake for a group of fifteen trace elements with generally scarce data and demonstrates that data from other countries, when available, are substantially similar. In addition, the estimated intakes are generally below the tolerable upper intake levels provided by international agencies. Thus, levels of trace elements in diet of the investigated population could be considered safe.

One of the strengths of our study is the collection and analysis of a large number of samples of foods and beverages that are actually purchased and consumed in the Emilia-Romagna Region. Secondly, we performed the estimation of dietary habits in a large population sample with overall dietary characteristics similar to those observed in other Italian populations (Agnoli et al. 2011; Malagoli et al. 2015). In addition, we used a detailed and validated food frequency questionnaire developed for the Northern Italian population (Pala et al. 2003; Pasanisi et al. 2002).

Our study also has some limitations which need to be acknowledged. We did not assess the bioavailability of trace elements after food ingestion by determining biological indicators of exposure, although previous studies suggested that only a fraction of total element intake is absorbed, especially in the presence of malabsorption disease (Reilly 2002). In addition, we did not carry out any speciation analysis for the selected elements, in spite of the increasing evidence of the importance of speciation analysis in the exposure assessment of trace elements possibly characterized by either toxicological and nutritional properties/features (Michalke et al. 2009, 2018; Ruzik 2012; Vinceti et al. 2017). Secondly, we did not differentiate between local and imported samples, hampering the ability to assess such a difference due to geographic origin of products. Finally, our study was carried out in adult population only, thus we could not evaluate dietary intake and safety levels in vulnerable population like children or pregnant women. With regard to some trace elements investigated, we did not evaluate the influence of other dietary sources of the metal such as cooking material and food containers, as well as other possible 'unconventional' dietary intake, e.g. toothpaste for titanium (Heringa et al. 2016; Perello et al. 2008; Ramos et al. 2016). Despite we limiting our assessment to dietary intake only, as the most relevant source in the general population (Reilly 2002), however, the evaluation of overall exposure in humans requires that additional sources be considered, particularly use of dietary supplements (which may represent the highest source of such trace elements in high consumers), dermal contact or air pollution (Rautiainen et al. 2016; Reilly 2002; WHO 1996). Dietary supplements consumption, however, is unlikely to have induced any substantial exposure misclassification in our investigation, since their use was very rare in the study population (Vinceti et al. 2011), as generally occurring in the Italian population.

Acknowledgements This work was supported by the and Reggio Emilia Local Health Authority of the National Health Service.

\section{Compliance with Ethical Standards}

Conflict of interest The authors declare that they have no conflict of interest.

Open Access This article is distributed under the terms of the Creative Commons Attribution 4.0 International License (http://creativeco mmons.org/licenses/by/4.0/), which permits unrestricted use, distribution, and reproduction in any medium, provided you give appropriate credit to the original author(s) and the source, provide a link to the Creative Commons license, and indicate if changes were made.

\section{References}

Adel M, Oliveri Conti G, Dadar M, Mahjoub M, Copat C, Ferrante M (2016) Heavy metal concentrations in edible muscle of whitecheek shark, Carcharhinus dussumieri (elasmobranchii, chondrichthyes) from the Persian Gulf: a food safety issue. Food Chem Toxicol 97:135-140. https://doi.org/10.1016/j.fct.2016.09.002

Agnoli C et al (2011) A priori-defined dietary patterns are associated with reduced risk of stroke in a large Italian cohort. J Nutr 141:1552-1558. https://doi.org/10.3945/jn.111.140061

Alberti-Fidanza A, Burini G, Perriello G (2002) Trace elements in foods and meals consumed by students attending the faculty cafeteria. Sci Total Environ 287:133-140. https://doi.org/10.1016/ S0048-9697(01)01009-9

Anke M, Seeber O, Müller R, Schäfer U, Zerull J (2009) Uranium transfer in the food chain from soil to plants, animals and man. Geochemistry 69:75-90. https://doi.org/10.1016/j.cheme r.2007.12.001

ANSES (2011) French Agency for Food, Environmental and Occupational Health \& Safety, 2011. Second French Total Diet Study

Arnich N, Sirot V, Riviere G, Jean J, Noel L, Guerin T, Leblanc JC (2012) Dietary exposure to trace elements and health risk assessment in the 2nd French Total Diet Study. Food Chem Toxicol 50:2432-2449. https://doi.org/10.1016/j.fct.2012.04.016

ATSDR (2007) Toxicological profile for barium. Agency for Toxic Substances and Disease Registry, Atlanta

Bachler G, von Goetz N, Hungerbuhler K (2015) Using physiologically based pharmacokinetic (PBPK) modeling for dietary risk assessment of titanium dioxide $\left(\mathrm{TiO}_{2}\right)$ nanoparticles. Nanotoxicology 9:373-380. https://doi.org/10.3109/17435390.2014.940404

Biego GH, Joyeux M, Hartemann P, Debry G (1998) Daily intake of essential minerals and metallic micropollutants from foods in France. Sci Total Environ 217:27-36. https://doi.org/10.1016/ S0048-9697(98)00160-0

Bocio A, Nadal M, Domingo JL (2005) Human exposure to metals through the diet in Tarragona, Spain: temporal trend. Biol Trace Elem Res 104:193-201. https://doi.org/10.1385/bter:104:3:193

Bonsignore $\mathrm{M}$ et al (2018) Bioaccumulation of heavy metals in fish, crustaceans, molluscs and echinoderms from the Tuscany coast. Ecotoxicol Environ Saf 162:554-562. https://doi.org/10.1016/j. ecoenv.2018.07.044 
Bruce RM, Odin M, WHO, International Programme on Chemical Safety (2001) Concise international chemical assessment document 32. Beryllium and beryllium compounds. WHO, Geneva

Copat C et al (2018) Trace elements in seafood from the Mediterranean sea: an exposure risk assessment. Food Chem Toxicol 115:13-19. https://doi.org/10.1016/j.fct.2018.03.001

Dolara P (2014) Occurrence, exposure, effects, recommended intake and possible dietary use of selected trace compounds (aluminium, bismuth, cobalt, gold, lithium, nickel, silver). Int $\mathbf{J}$ Food Sci Nutr 65:911-924. https://doi.org/10.3109/09637 486.2014.937801

Domingo JL, Perello G, Gine Bordonaba J (2012) Dietary intake of metals by the population of Tarragona County (Catalonia, Spain): results from a duplicate diet study. Biol Trace Elem Res 146:420-425. https://doi.org/10.1007/s12011-011-9269-5

ECHA (2008) European Union risk assessment report: diantimony trioxide CAS No.: 1309-64-4. European Commission's Joint Research Centre. Office for Official Publications of the European Communities, Luxembourg

European Food Safety Authority (2004a) Opinion of the scientific panel on dietetic products, nutrition and allergies on a request from the commission related to the tolerable upper intake level of vanadium. EFSA J 33:1-22. https://doi.org/10.2903/j.efsa.2009.1084

European Food Safety Authority (2004b) Opinion of the scientific panel on food additives, flavourings, processing aids and materials in contact with food (AFC) on a request from the Commission related to a 2 nd list of substances for food contact materials. EFSA J 24:1-13. https://doi.org/10.2903/j.efsa.2004.24a

European Food Safety Authority (2004c) Opinion of the scientific panel on dietetic products, nutrition and allergies on a request from the commission related to the tolerable upper intake level of boron (sodium borate and boric acid). EFSA J 80:1-22. https ://doi.org/10.2903/j.efsa.2004.80

European Food Safety Authority (2006) Tolerable upper intake levels for vitamins and minerals. European Food Safety Authority, Parma

European Food Safety Authority (2009a) Inability to assess the safety of lithium-enriched yeast added for nutritional purposes as a source of lithium in food supplements and the bioavailability of lithium from this source, based on the supporting dossier. EFSA J 1086:1-6. https://doi.org/10.2903/j.efsa.2009.1086

European Food Safety Authority (2009b) Uranium in foodstuffs, in particular mineral water. EFSA J 1018:1-59. https://doi. org/10.2903/j.efsa.2009.1018

European Food Safety Authority (2009c) Inability to assess the safety of strontium-enriched yeast added for nutritional purposes as a source of strontium in food supplements and the bioavailability of strontium from this source, based on the supporting dossier. EFSA J. https://doi.org/10.2903/j.efsa.2009.1085

European Food Safety Authority (2011) Call for scientific data on Silver (E 174) and Gold (E 175), used as food colours. https://www. efsa.europa.eu/en/consultations/call/110223

European Food Safety Authority (2012) EFSA Panel on Additives and Products or Substances used in Animal Feed (FEEDAP): scientific opinion on safety and efficacy of cobalt compounds (E3) as feed additives for all animal species: Cobaltous acetate tetrahydrate, basic cobaltous carbonate monohydrate and cobaltous sulphate heptahydrate, based on a dossier submitted by TREAC EEIG. EFSA J 10:2971. https://doi.org/10.2903/j.efsa.2012.2791

European Food Safety Authority (2013) Scientific opinion on dietary reference values for molybdenum. EFSA J 11:3333. https://doi. org/10.2903/j.efsa.2013.3333

European Food Safety Authority (2015) Scientific Opinion on the risks to public health related to the presence of nickel in food and drinking water. EFSA J 13:4002. https://doi.org/10.2903/j. efsa.2015.4002
European Food Safety Authority (2016a) Re-evaluation of titanium dioxide (E 171) as a food additive. EFSA J 14:4545. https://doi. org/10.2903/j.efsa.2016.4545

European Food Safety Authority (2016b) Scientific opinion on the reevaluation of silver (E 174) as food additive. EFSA J 14:4364. https://doi.org/10.2903/j.efsa.2016.4364

European Food Safety Authority (2018) Evaluation of four new studies on the potential toxicity of titanium dioxide used as a food additive (E 171). EFSA J 16:5366. https://doi.org/10.2903/j. efsa.2018.5366

European Food Safety Authority (2019) EFSA statement on the review of the risks related to the exposure to the food additive titanium dioxide (E 171) performed by the French Agency for Food, Environmental and Occupational Health and Safety (ANSES). EFSA J 17:5714. https://doi.org/10.2903/j.efsa.2019.5714

Evans WH, Read JI, Caughlin D (1985) Quantification of results for estimating elemental dietary intakes of lithium, rubidium, strontium, molybdenum, vanadium and silver. Anal 110:873877. https://doi.org/10.1039/an9851000873

FAO/WHO (1969) Toxicological evaluation of some food colours, emulsifiers, stabilizers, anti-caking agents and certain other substances: titanium dioxide. FAO nutrition meeting report series, vol 46A. WHO, Geneva

Ferrante M et al (2018) PAHs in seafood from the Mediterranean Sea: an exposure risk assessment. Food Chem Toxicol 115:385-390. https://doi.org/10.1016/j.fct.2018.03.024

Filippini T et al (2017a) Toenail selenium as an indicator of environmental exposure: a cross-sectional study. Mol Med Rep 15:34053412. https://doi.org/10.3892/mmr.2017.6388

Filippini T et al (2017b) Dietary determinants of serum selenium species in Italian populations. J Trace Elem Med Biol 41S:39. https ://doi.org/10.1016/j.temb.2017.03.163

Filippini T et al (2018a) Dietary intake of cadmium, chromium, copper, manganese, selenium and zinc in a Northern Italy community. J Trace Elem Med Biol 50:508-517. https://doi.org/10.1016/j. jtemb.2018.03.001

Filippini $\mathrm{T}$ et al (2018b) Intake of arsenic and mercury from fish and seafood in a Northern Italy community. Food Chem Toxicol 116:20-26. https://doi.org/10.1016/j.fct.2018.04.010

Filippini T, Malagoli C, Wise LA, Malavolti M, Pellacani G, Vinceti M (2019a) Dietary cadmium intake and risk of cutaneous melanoma: an Italian population-based case-control study. J Trace Elem Med Biol 56:100-106. https://doi.org/10.1016/j.jtemb .2019 .08 .002

Filippini T et al (2019b) Aluminum and tin: Food contamination and dietary intake in an Italian population. J Trace Elem Med Biol 52:293-301. https://doi.org/10.1016/j.jtemb.2019.01.012

Finley BL, Monnot AD, Paustenbach DJ, Gaffney SH (2012) Derivation of a chronic oral reference dose for cobalt. Regul Toxicol Pharmacol 64:491-503. https://doi.org/10.1016/j.yrtph .2012.08.022

Gambelli L, Belloni P, Ingrao G, Pizzoferrato L, Santaroni GP (1999) Minerals and trace elements in some Italian dairy products. J Food Compos Anal 12:27-35. https://doi.org/10.1006/ jfca.1998.0802

German Federal Institute for Risk Assessment (BfR) (2004) Thallium in natürlichem Mineralwasser

Gibson RS, Scythes CA (1984) Chromium, selenium, and other trace element intakes of a selected sample of Canadian premenopausal women. Biol Trace Elem Res 6:105-116. https://doi.org/10.1007/ bf02916928

Gimou MM, Pouillot R, Charrondiere UR, Noel L, Guerin T, Leblanc JC (2014) Dietary exposure and health risk assessment for 14 toxic and essential trace elements in Yaounde: the Cameroonian total diet study. Food Addit Contam Part A Chem Anal Control 
Expo Risk Assess 31:1064-1080. https://doi.org/10.1080/19440 049.2014.909953

Gonzalez-Weller D et al (2013) Dietary intake of barium, bismuth, chromium, lithium, and strontium in a Spanish population (Canary Islands, Spain). Food Chem Toxicol 62:856-858. https ://doi.org/10.1016/j.fct.2013.10.026

Heringa MB, Geraets L, van Eijkeren JC, Vandebriel RJ, de Jong WH, Oomen AG (2016) Risk assessment of titanium dioxide nanoparticles via oral exposure, including toxicokinetic considerations. Nanotoxicology 10:1515-1525. https://doi.org/10.1080/17435 390.2016 .1238113

Hunt CD, Meacham SL (2001) Aluminum, boron, calcium, copper, iron, magnesium, manganese, molybdenum, phosphorus, potassium, sodium, and zinc: concentrations in common western foods and estimated daily intakes by infants; toddlers; and male and female adolescents, adults, and seniors in the United States. J Am Diet Assoc 101:1058-1060. https://doi.org/10.1016/s0002 -8223(01)00260-7

IARC (1990) Chromium, nickel and welding IARC Monographs on the evaluation of carcinogenic risks to humans 49

Institute of Medicine (2001) Dietary reference intakes for vitamin a, vitamin $\mathrm{k}$, arsenic, boron, chromium, copper, iodine, iron, manganese, molybdenum, nickel, silicon, vanadium, and zinc. National Academy Press, Washington, D.C.

IRIS Assessments (2019). https://cfpub.epa.gov/ncea/iris_drafts/AtoZ. cfm. Accessed 10 July 2019

Iyengar GV, Wolfe WR, Tanner JT, Morris ER (2000) Content of minor and trace elements, and organic nutrients in representative mixed total diet composites from the USA. Sci Total Environ 256:215226. https://doi.org/10.1016/S0048-9697(00)00494-0

Kim JH, Gibb HJ, Howe PD, WHO, Chemical Safety Team \& International Programme on Chemical Safety (2006) Cobalt and inorganic cobalt compounds/prepared by James H. Kim, Herman J. Gibb, Paul D. Howe. WHO, Geneva

Kim CI, Lee J, Kwon S, Yoon HJ (2015) Total diet study: for a closerto-real estimate of dietary exposure to chemical substances. Toxicol Res 31:227-240. https://doi.org/10.5487/TR.2015.31.3.227

Kron T, Hansen C, Wernert E (1991) Tellurium ingestion with foodstuffs. J Food Compos Anal 4:196-205. https://doi. org/10.1016/0889-1575(91)90031-Z

Larsen EH, Andersen NL, Moller A, Petersen A, Mortensen GK, Petersen J (2002) Monitoring the content and intake of trace elements from food in Denmark. Food Addit Contam 19:33-46. https://doi.org/10.1080/02652030110087447

Leblanc JC, Guerin T, Noel L, Calamassi-Tran G, Volatier JL, Verger P (2005) Dietary exposure estimates of 18 elements from the 1st French Total Diet Study. Food Addit Contam 22:624-641. https ://doi.org/10.1080/02652030500135367

Llobet JM, Granero S, Schuhmacher M, Corbella J, Domingo JL (1998) Biological monitoring of environmental pollution and human exposure to metals in Tarragona, Spain. IV. Estimation of the dietary intake. Trace Elem Electrolytes 15:136-141

Malagoli C et al (2015) Diet quality and risk of melanoma in an Italian population. J Nutr 145:1800-1807. https://doi.org/10.3945/ jn.114.209320

Malagoli C, Malavolti M, Farnetani F, Longo C, Filippini T, Pellacani G, Vinceti M (2019) Food and beverage consumption and melanoma risk: a population-based case-control study in Northern Italy. Nutrients 11:2206. https://doi.org/10.3390/nu11092206

Malavolti M et al (2013) Association between dietary vitamin C and risk of cutaneous melanoma in a population of Northern Italy. Int J Vitam Nutr Res 83:291-298. https://doi.org/10.1024/03009831/a000171

Malavolti M et al (2017) Glycaemic index, glycaemic load and risk of cutaneous melanoma in a population-based, case-control study.
Br J Nutr 117:432-438. https://doi.org/10.1017/s000711451 $700006 \mathrm{x}$

Marcussen H, Jensen BH, Petersen A, Holm PE (2013) Dietary exposure to essential and potentially toxic elements for the population of Hanoi, Vietnam. Asia Pac J Clin Nutr 22:300-311. https://doi. org/10.6133/apjen.2013.22.2.06

Meacham SL, Hunt CD (1998) Dietary boron intakes of selected populations in the United States. Biol Trace Elem Res 66:65-78. https ://doi.org/10.1007/bf02783127

Michalke B, Halbach S, Nischwitz V (2009) JEM spotlight: metal speciation related to neurotoxicity in humans. J Environ Monit 11:939-954. https://doi.org/10.1039/b817817h

Michalke B, Willkommena D, Drobyshevb E, Solovyev N (2018) The importance of speciation analysis in neurodegeneration research. Trends Analyt Chem 104:160-170. https://doi.org/10.1016/j. trac.2017.08.008

Mitchell PB, Hadzi-Pavlovic D (2000) Lithium treatment for bipolar disorder. Bull World Health Organ 78:515-517

Nielsen FH (2014) Update on human health effects of boron. J Trace Elem Med Biol 28:383-387. https://doi.org/10.1016/j.jtemb .2014 .06 .023

Noel L, Leblanc JC, Guerin T (2003) Determination of several elements in duplicate meals from catering establishments using closed vessel microwave digestion with inductively coupled plasma mass spectrometry detection: estimation of daily dietary intake. Food Addit Contam 20:44-56. https://doi.org/10.1080/0265203021 000031573

Nordberg M, Nordberg GF (2016) Trace element research-historical and future aspects. J Trace Elem Med Biol 38:46-52. https://doi. org/10.1016/j.jtemb.2016.04.006

Ozgur S, Sumer H, Kocoglu G (1996) Rickets and soil strontium. Arch Dis Child 75:524-526. https://doi.org/10.1136/adc.75.6.524

Pala V et al (2003) Diet in the Italian EPIC cohorts: presentation of data and methodological issues. Tumori 89:594-607

Pasanisi P, Berrino F, Bellati C, Sieri S, Krogh V (2002) Validity of the Italian EPIC questionnaire to assess past diet. IARC Sci Publ $156: 41-44$

Pennington JA, Jones JW (1987) Molybdenum, nickel, cobalt, vanadium, and strontium in total diets. J Am Diet Assoc 87:1644-1650

Perello G, Marti-Cid R, Llobet JM, Domingo JL (2008) Effects of various cooking processes on the concentrations of arsenic, cadmium, mercury, and lead in foods. J Agric Food Chem 56:11262-11269. https://doi.org/10.1021/jf802411q

Rainey C, Nyquist L (1998) Multicountry estimation of dietary boron intake. Biol Trace Elem Res 66:79-86. https://doi.org/10.1007/ bf02783128

Ramos K, Gomez-Gomez MM, Camara C, Ramos L (2016) Silver speciation and characterization of nanoparticles released from plastic food containers by single particle ICPMS. Talanta 151:83-90. https://doi.org/10.1016/j.talanta.2015.12.071

Rautiainen S, Manson JE, Lichtenstein AH, Sesso HD (2016) Dietary supplements and disease prevention: a global overview. Nat Rev Endocrinol 12:407-420. https://doi.org/10.1038/nrendo.2016.54

Reilly C (2002) Metal contamination of food: its significance for food quality and human health. Blackwell, Oxford. https://doi. org/10.1002/9780470995105

Rompelberg C et al (2016) Oral intake of added titanium dioxide and its nanofraction from food products, food supplements and toothpaste by the Dutch population. Nanotoxicology 10:1404-1414. https://doi.org/10.1080/17435390.2016.1222457

Rose M, Baxter M, Brereton N, Baskaran C (2010) Dietary exposure to metals and other elements in the 2006 UK Total Diet Study and some trends over the last 30 years. Food Addit Contam Part A Chem Anal Control Expo Risk Assess 27:1380-1404. https:// doi.org/10.1080/19440049.2010.496794 
Ruzik L (2012) Speciation of challenging elements in food by atomic spectrometry. Talanta 93:18-31. https://doi.org/10.1016/j.talan ta.2012.01.066

Santos EE, Lauria DC, Porto da Silveira CL (2004) Assessment of daily intake of trace elements due to consumption of foodstuffs by adult inhabitants of Rio de Janeiro city. Sci Total Environ 327:69-79. https://doi.org/10.1016/j.scitotenv.2004.01.016

SCHER (2012) Assessment of the tolerable daily intake of barium. European Union, London

Schrauzer GN (2002) Lithium: occurrence, dietary intakes, nutritional essentiality. J Am Coll Nutr 21:14-21. https://doi. org/10.1080/07315724.2002.10719188

Schroeder HA, Buckman J, Balassa JJ (1967) Abnormal trace elements in man: tellurium. J Chron Dis 20:147-161. https://doi. org/10.1016/0021-9681(67)90049-5

Shimbo S et al (1996) Use of a food composition database to estimate daily dietary intake of nutrient or trace elements in Japan, with reference to its limitation. Food Addit Contam 13:775-786. https ://doi.org/10.1080/02652039609374465

Tiesjema B, Baars AJ (2009) Re-evaluation of some human-toxicological maximum permissible risk levels earlier evaluated in the period 1991-2001 vol RIVM Report 711701092/2009. National Institute for Public Health and the Environment, Bilthoven, the Netherlands

Turconi G, Minoia C, Ronchi A, Roggi C (2009) Dietary exposure estimates of twenty-one trace elements from a Total Diet Study carried out in Pavia, Northern Italy. Br J Nutr 101:1200-1208. https://doi.org/10.1017/S0007114508055670

Turrini A, Saba A, Perrone D, Cialfa E, D’Amicis A (2001) Food consumption patterns in Italy: the INN-CA Study 1994-1996. Eur J Clin Nutr 55:571-588. https://doi.org/10.1038/sj.ejen.1601185

Vinceti M et al (2011) Inverse association between dietary vitamin $\mathrm{D}$ and risk of cutaneous melanoma in a Northern Italy population. Nutr Cancer 63:506-513. https://doi.org/10.1080/01635 581.2011.539314

Vinceti M et al (2017) A selenium species in cerebrospinal fluid predicts conversion to Alzheimer's dementia in persons with mild cognitive impairment. Alzheimers Res Ther 9:100. https://doi. org/10.1186/s13195-017-0323-1

Wappelhorst O, Kuhn I, Heidenreich H, Markert B (2002) Transfer of selected elements from food into human milk. Nutrition 18:316322. https://doi.org/10.1016/S0899-9007(01)00798-5

Weir A, Westerhoff P, Fabricius L, Hristovski K, von Goetz N (2012) Titanium dioxide nanoparticles in food and personal care products. Environ Sci Technol 46:2242-2250. https://doi. org/10.1021/es204168d

WHO (1996) Trace elements in human health and nutrition. World Health Organization, Geneva

WHO (2000) Chapter 6.12 vanadium. WHO Regional Office for Europe, Copenhagen, Denmark

WHO (2003a) Molybdenum in drinking water, background document for preparation of WHO guidelines for drinking water quality. WHO Press, Geneva

WHO (2003b) Antimony in drinking water, background document for preparation of WHO guidelines for drinking water quality. WHO Press, Geneva

WHO (2003c) Silver in drinking water, background document for preparation of WHO guidelines for drinking water quality. WHO Press, Geneva

WHO (2003d) Boron in drinking-water background document for development of WHO guidelines for drinking-water quality. WHO Press, Geneva

WHO (2004) Guidelines for drinking-water quality, 3rd edn. WHO Press, Geneva

WHO (2005) Nickel in drinking-water. Background document for development of WHO Guidelines for drinking-water quality. WHO Press, Geneva

WHO (2009) Beryllium in drinking-water. Background document for development of WHO guidelines for drinking-water quality. WHO Press, Geneva

WHO (2010) Strontium and strontium compunds. International Programme on Chemical Safety \& Inter-Organization Programme for the Sound Management of Chemicals. WHO Press, Geneva

WHO (2016) Barium in Drinking-water. Background document for development of WHO Guidelines for Drinking-water Quality. WHO Press, Geneva

WHO, International Programme for Chemical Safety (1996) Thallium. Environmental health criteria 182, Geneva

Winkler HC, Notter T, Meyer U, Naegeli H (2018) Critical review of the safety assessment of titanium dioxide additives in food. J Nanobiotechnol 16:51. https://doi.org/10.1186/s1295 1-018-0376-8

Ysart G et al (1999) Dietary exposure estimates of 30 elements from the UK Total Diet Study. Food Addit Contam 16:391-403. https ://doi.org/10.1080/026520399283876

Publisher's Note Springer Nature remains neutral with regard to jurisdictional claims in published maps and institutional affiliations. 